\title{
Jean Lambert, L'Ancienne exégèse et la Nouvelle
}

Jean Lambert

\section{(2) OpenEdition}

\section{Journals}

Édition électronique

URL : http://journals.openedition.org/assr/24565

DOI : $10.4000 /$ assr. 24565

ISSN : $1777-5825$

Éditeur

Éditions de l'EHESS

Édition imprimée

Date de publication : 30 décembre 2012

Pagination : 75-89

ISSN : 0335-5985

\section{Référence électronique}

Jean Lambert, "Jean Lambert, L'Ancienne exégèse et la Nouvelle 》, Archives de sciences sociales des religions [En ligne], 160 | octobre-décembre 2012, mis en ligne le 20 février 2016, consulté le 19 avril 2019. URL : http://journals.openedition.org/assr/24565 ; DOI : 10.4000/assr.24565 
puis sous celle d'un film hollywoodien. La dimension scandaleuse de l'affaire, pour l'Église et pour beaucoup de croyants tient à la façon dont ce roman pseudohistorique exploite l'espace de non-conformisme et de liberté ouvert par la critique biblique. On peut y voir à la fois l'apothéose et la damnation de la recherche bibliste, dans sa dimension de liberté et de distanciation par rapport à l'approche théologique et dogmatique traditionnelle.

En conclusion, l'ouvrage pose la question de la place de la recherche bibliste à l'intérieur des sciences humaines, en particulier les sciences philologiques et textuelles, dont le contenu semble se renouveler sous l'influence de l'approche narratologique. Également de leur place dans le champ qui pourrait se dessiner autour d'une approche comparative des grands Textes canoniques : Classiques chinois, Textes de la Révélation védique et hindoue, Textes bouddhistes, Coran et Hadith... Quels seraient les effets scientifiques, culturels, idéologiques et politiques d'une telle approche comparative, où l'exégèse biblique perdrait son droit d'exterritorialité, pour être mobilisée en tant que champ particulier d'une telle approche ? Les questions bibliques avaient été dominées au XIX et au XX ${ }^{\mathrm{e}}$ siècle par la question de la légitimation d'un nouveau mode de lecture - historique et philologique - de la Bible. L'originalité du travail de Pierre Lassave réside dans l'exercice d'un nouveau type de questionnement, caractérisé par une approche sociologique et ethnographique du monde des lecteurs professionnels de la Bible répondant à l'appel du Texte.

Gérard LECLERC

Université Paris VIII gerard.lec@wanadoo.fr

Jean Lambert

\section{L'Ancienne exégèse et la Nouvelle}

"Tout texte est le tissu de sa genèse " (Michel Serres)

Cette enquête sur des biblistes chrétiens se présente comme une sociologie paradoxale d'un savoir conséquent (et fort ancien), au carrefour de disciplines attestées, où se déploie un pluralisme irréductible de significations pour un objet partagé sinon unique. L'ethnographie de ce milieu composite est pionnière, et bienvenue en ces temps de regain d'intérêt pour le religieux. Elle interroge la nature de ce savoir à travers ses manuels (composition du Pentateuque, quête du Jésus historique : comment progresse l'histoire des mythes ?), ses institutions (les biblistes sont-ils des scientifiques ?), ses colloques (conflits d'interprétations, 
pathos contre logos ?), ses acteurs (biographies de chrétiens déviants ?), et ses échos médiatiques (Corpus Christi, Da Vinci code, l'Évangile de Judas). Le succès de l'émission d'Arte, Corpus Christi, a conduit le sociologue à donner cette suite à son enquête sur la traduction littéraire de la bible chez Bayard (20012005). Elle découvre un monde de biblistes sans attaches, rajeuni, féminisé et laïcisé, qui pratiquent leur "petite science conjecturale " (Renan) pour un public lettré accru. L'enquête combine une information sur l'histoire de l'exégèse (surtout la perception de deux chantiers complexes sur l'Ancien et le Nouveau Testament), avec trois prélèvements : une esquisse des réseaux et des publics, la relation amusée de trois colloques, et la biographie existentielle de sept biblistes. Le dernier chapitre associe trois objets hétérogènes : la réception d'une vulgarisation télévisée, sagace et orientée, du travail exégétique ; un roman-farce commercial distrayant; les tribulations d'un manuscrit gnostique qui faillit échapper à l'édition scientifique. L'enquête a valeur de bilan pour un horizon qui s'achève. Elle semble conclure implicitement que les lectures de la Bible ne peuvent être autres que plurielles et de statut varié parce que les interprètes constituent un conglomérat disparate de compétences disciplinaires hétérogènes, produisant un savoir flou, dépendant de la croyance autant que de la science, mobilisé à une fin somme toute apologétique, l'appel du texte.

\section{"L'écriture progresse avec ceux qui la lisent " 1}

C'est à juste titre que Pierre Lassave procède par fragments, car le religieux est un système de miettes, mais il ne semble pas aller au bout de son intuition sur le caractère fractal de l'objet ou de ses lecteurs. Ce monde des biblistes étonne certes, parce qu'il partage le sort général du religieux de nos jours : morcelé, disparate; en conflits de décompositions/recompositions; à la jointure de sciences éprouvées et de lourds héritages politico-historiques; tiré entre des appartenances confessionnelles et des outsiders novateurs, etc., comme le sont les traditions, elles-mêmes méconnaissables aujourd'hui.

\section{Littérature ou histoire $=$ historico-critique ?}

D'un ouvrage foisonnant aux multiples angles d'approche, suivons la piste de l'historico-critique. Ces textes sacrés sont réputés depuis longtemps relever ni de simple littérature de fiction, ni d'histoire seulement, mais au minimum de

1. Scriptura cum legentibus crescit. Formule de Grégoire Le Grand qu'aimait citer Paul Ricœur, par exemple : in Penser la Bible (co-écrit avec André Lacocque), Paris, Le Seuil, coll. "Points-Essais » (1998), 2003, p. 442 où il adopte le subjonctif crescat comme pour mieux marquer un souhait. 
mélanges. D'où la formation de ce mixte méthodologique, critique littéraire et critique historique mêlées, que désigne en 1933 l'historico-critique ${ }^{2}$, qui se forme

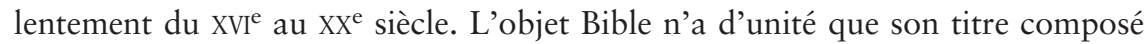
tardivement, couvrant une bibliothèque disparate aux contours toujours contestés et sans cesse élargis. Le philologue Éric Auerbach, dans Mimésis que cite l'auteur, a résolu la question pour 1946 : l'Illiade et la Bible forment deux styles. L'un décrit les événements en les extériorisant, sans arrière-fond caché, il appelle l'analyse. L'autre met en valeur certains éléments pour en laisser d'autres dans l'ombre, inexprimés, il appelle l'interprétation. Et comme les textes bibliques prétendaient seuls à l'autorité, l'interprétation en vient à englober la réalité extérieure, et "devient la méthode générale d'appréhension de la réalité » (Mimésis, p. 25). Ce qui se reproduit lorsque comme ici, Bible désigne à la fois Ancien et Nouveau Testament, ce qui est un usage chrétien (différent selon les catholiques où il y a substitution, et les protestants où il y a continuité), non conforme à la réalité des corpus. L'auteur, qui dit ne pas avoir de pratique effective de l'exégèse, accomplit le tour d'horizon utile d'un état dépassé de la science exégétique, sans pourtant aller jusqu'à caractériser les lignes de fuite qui s'ouvrent. Des biblistes eux-mêmes déplorent ne pas trouver un savoir classique aux contours scientifiques nets et en progrès là où, davantage qu'un savoir, il s'agit comme pour beaucoup de sciences aujourd'hui, d'un ensemble de pratiques qui se modifient.

\section{De l'exégèse à l'anthropologie}

Ces pratiques sont bien sûr duelles comme le sont toujours les pratiques (Michel de Certeau), certaines canoniques et réglées, usages reconnus et partagés, d'autres pirates, ruses créatrices et chemins de traverse. Elles dessinent le contour d'un milieu - ou plutôt de milieux aux frontières poreuses - qui, et c'est la nouveauté, sort sans cesse de sa discipline, de ses disciplines, fictions comprises, pour y revenir en juxtaposant/combinant intertextualité narrative et sémiotique littéraire, informations archéologiques, érudition philologique, synthèses anthropologiques, herméneutique esthétique, philosophies critiques, et bien sûr des théologies rémanentes, parmi une enquête historique entêtée. Ce milieu combine, enchaîne par à-coups ou en glissant, bref il mixe, comme jouerait un DJ théoricien, ou comme le faisaient jadis Vladimir Jankélévitch et naguère Gilles Deleuze, ou comme pourrait le penser un géologue dynamicien d'un paysage atlasique complexe, marécage issu de plaques tectoniques divisées, élevé, érodé, sédimenté, raviné, coupé de failles, etc. Cette nouvelle manière de pratiquer de la science,

2. A. Rey, Dictionnaire historique de la langue française, 2010. L'expression Historischkritisch serait due à Johann Salomo Semler (1725-1791); devenue d'usage courant au XIX siècle allemand, les Grimm en signalent l'emploi par Goethe, $c f$. F. Laplanche, La Bible en France, entre mythe et critique, XVI ${ }^{e}$ XIX ${ }^{e}$ siècle, Paris, Albin Michel, coll. "L'évolution de l'humanité ", 1994, p. 11, no 3. 
et la science des mythes en particulier, de la science humaine unifiée par un style d'anthropologie à la française, nourrie de philosophie et de modèles littéraires, et non de pointillisme anglo-saxon, illustre la grande mutation des exégètes en anthropologues, c'est-à-dire d'historiens-critiques qui quittent le statut d'incomparabilité de leur espace (texte, langue, dieu, ethnie ou sens) dont ils ont abusé ou qui les a abusés, pour des chemins de traverse désormais comparatifs. "Dès qu'un historien se propose de rendre compte de la variabilité des cultures humaines, il pense en anthropologue » (Marcel Detienne ${ }^{3}$ ).

\section{Comparer, enfin...}

La nouveauté des pratiques comparatives éclaire celle des acteurs. Savants exégètes (laïques) ou (pieux) biblistes vulgarisateurs, l'auteur cherche une distinction. Or de l'un à l'autre, et retour, la conséquence est bonne. Le texte fluctuant s'offre à des lecteurs aléatoires, savantissimes souvent, toujours érudits, infiniment curieux, qui vont vers la simplicité ou en viennent, selon des trajectoires fortes. De ces milieux héritiers de querelles canoniques antiques, médiévales, modernes, romantiques, positivistes (foi/raison) et disciplinaires (où tous les champs disciplinaires et les aires culturelles exhibent leurs théologiens, leurs légistes et leurs douaniers...) des exégètes s'extirpent (existentiellement et scripturairement, mais à quel prix...) en inventant des trouvailles atypiques et des modèles provisoires qui, à terme, seront porteurs de mutations lourdes. Un petit exemple récent : comment traduire le verset du Deutéronome 32, 8 - "Le TrèsHaut fixa le territoire des peuples suivant le nombre des fils d'Israël » - selon le texte massorétique? "[...] suivant le nombre des anges de Dieu » selon la Septante ? «[...] suivant le nombre des fils de Dieu, ou fils d'El » selon les manuscrits de Qumrân (4QDt) ? Jan Joosten à Strasbourg propose une solution ${ }^{4}$. Par regroupement des lettres et dittographie du yod, le passage hébraïque de beni$E l$ à beni-Israël se ferait par beni-sr-El (non attesté dans la Bible) qui désigne en ougaritique le Taureau El, père des dieux : "Baal crie vers le Taureau, El, son père (tr.il.abh), vers El, le roi, qui l'a créé » (CAT 1.3 V 35-36). Habile, mais les prêtres tardifs connaissaient-ils encore l'ougaritique... ? L'affaire rebondit.

\section{Ce que fait le texte avant son lecteur}

Il est certain que le texte est ce que fait le texte, qui est structuraliste avant son lecteur, et philologue, narratologue ou sémioticien avant son herméneute.

3. Préface au Livre de traverse, de l'exégèse biblique à l'anthropologie, Paris, Éditions du Cerf, coll. «Patrimoines », 1992, p. 11.

4. E. Bons, T. Legrand (dir.), Le monothéisme biblique, évolution, contextes et perspectives, Paris, Éditions du Cerf, coll. « Lectio divina », 2011, p. 101. 
C'est au lecteur de se porter au niveau des pratiques de l'œuvre qu'il étudie. Si on trouve telles oppositions distinctives, telles structures de l'espace et du temps, telles architectures et telles tensions de façon probante, c'est que le texte les active, donc les cultures dont il est issu. Georges Dumézil n'invente pas les trois fonctions dans les textes indo-européens, il les découvre parce que le texte compare avant lui. Et donc les littératures connexes, analogues ou opposées appartiennent au vaste champ d'investigation comparée, avec toutes les méthodes requises. L'exégèse anthropologique met en évidence des constantes différenciées des pratiques humaines (comment faire de la fondation, du pouvoir, de la loi, du territoire, du mythe, du rite, du salut ou du divin ?) pour des champs culturels différenciés où se construit petit à petit la grammaire contrastive des règles de production des cultures. Comment écrire de l'histoire en différents régimes d'bistoricité ? Quelles figures de l'événement permettent d'en comprendre les changements ? L'historico-critique, acquis durable et indispensable, permet à l'exégèse biblique de passer à l'anthropologie. L'auteur observe une information érudite qui progresse (crescat!) très rapidement en rhizomes, dont les chantiers sont sans cesse rouverts ou déplacés, où les questions fortes du XX siècle (mythe, histoire et interprétation) n'ont plus le même poids, tandis que les hypothèses pullulent. Le corpus s'élargit d'apocryphes ou d'intertestamentaires, de langues (ougaritique), d'archéologie (Qûmran), d'entours historiques et de révisions majeures. Mais aperçoit-il les neuves comparaisons enfin commencées avec des espaces culturels connexes, ou au contraire contrastés ? Cet ensemble de sciences et d'hypothèses mute et déforme sans cesse l'objet incertain et accru auquel elles s'appliquent. Au point que la sociologie de l'exégèse pourrait devenir une agnotologie, une science de l'ignorance élaborée par des sociétés pour (se) masquer le fruit de leurs découvertes comparées... et y résister par des institutions propres !

\section{La rupture instauratrice}

Michel de Certeau, en pionnier de l'épistémologie de l'exégèse - mais il courait déjà ailleurs -, avait ouvert l'horizon dans Lieux de transit (revue Esprit, 1973) - texte dont manque la première version plus longue, réuni au lendemain de sa mort par Luce Giard dans La faiblesse de croire (Le Seuil, 1987). Il constatait une fin du religieux, aux besoins duquel répondraient pour une part les sciences humaines... À l'intérieur de ce moment, il remarquait le repli défensif et réflexe de l'exégèse sur sa forteresse, incapable de penser «la rupture instauratrice " (décrite dans son projet de thèse en théologie de 1971). Tout était annoncé $d u$ glissement de l'exégèse vers l'anthropologie. Glissement institutionnel aussi, hors des lieux auscultés par l'auteur, dont par exemple Françoise SmythFlorentin, Marcel Detienne et ceux qu'ils rassemblent dans une large ATPCNRS comparative illustrent les avancées (Tracés de fondation, 1990 ; Le livre de traverse, 1992 ; Transcrire les mythologies, 1994 ; "Pour un débat sur les 
historicités comparées ", $1996^{5}$ ). Dans ce dépassement de l'historico-critique, accompagné de la menace des littéralismes, Certeau voyait la fin des espaces confessionnels distincts, et partant des espaces canoniques des textes, comme il percevait indues les séparations des disciplines. L'auteur qui cite souvent ce texte de 1973 ne semble en retenir que le souvenir de la formule : " une technique impressionnante et une épistémologie déficiente " (La faiblesse de croire, p. 240) qui caractérise les deux congrès observés, Claremont et Los Angeles. Or dans L'invention du quotidien 1, Arts de faire (1980) Certeau précise la rupture de l'historien-anthropologue (p. 153) : «Marcel Detienne a délibérément choisi de raconter. Il n'installe pas les histoires grecques devant lui pour les traiter au nom d'autre chose qu'elles-mêmes. Il refuse la coupure qui en ferait des objets de savoir, mais aussi des objets à savoir, cavernes où des "mystères" en réserve attendraient de l'investigation scientifique leur signification. Il ne suppose pas, derrière toutes ces histoires, des secrets dont le progressif dévoilement lui donnerait, en retrait, sa propre place, celle de l'interprétation. [...] Vous demandez ce qu'ils "veulent" dire ? Je vais vous les raconter à nouveau. À qui l'interrogeait sur le sens d'une sonate, Beethoven, dit-on, la rejouait ». Certeau décrit la nouvelle pratique de l'exégèse.

En résumé, oublions Ratzinger rejetant l'historico-critique en 2011 comme " un cimetière d'hypothèses contradictoires ", quand celle-ci sort depuis quarante ans du champ comme approche dominante, depuis les vues prédictives de Certeau de 1971. Detienne et bien d'autres ont pris acte en vingt ans du glissement de l'exégèse dans l'anthropologie comparative, et dès lors le territoire biblique est tout sauf réputé incomparable dans les laboratoires ou parmi les chercheurs, depuis que le fait religieux textuel - «la mythologie ça s'écrit » (M. Detienne, L'invention de la mythologie, 1981) - n'est qu'un cas particulier du fait culturel. Un artefact trop français continue d'écarter la Bible de la littérature générale étudiée à l'université, tandis que les faits religieux chrétiens - donc les textes - tardent à accéder peu à peu au champ anthropologique, et qu'y parviennent lentement faits musulmans et juifs, chacun selon sa distance supposée d'avec le fait chrétien. Que par ailleurs ce texte, comme d'autres en provenance d'autres cultures, appelle (pour reprendre le titre bergsonien de l'ouvrage) tel lecteur comme individu en quête de foyer spirituel, c'est une autre affaire, existentielle, qui relève de l'étude de la croyance. Pascal, pour avoir vécu sa nuit de feu, est-il moins inventeur des probabilités, du placard, d'une exégèse biblique, de la brouette ou des transports en commun, et on le sait maintenant, de l'ordinateur?

5. A. de Pury, T. Römer, J.-D. Macchi (dirs.), Israël construit son histoire, Genève, Labor et Fides, coll. «Le monde de la Bible », 1996, p. 153-166. 


\section{Une gnose nomade}

Comme tout commentaire second, la sociologie risque parfois d'acquérir le statut d'une gnose par rapport à l'exégèse, c'est-à-dire d'une sorte de savoir englobant issu d'un point de vue spécifique et de pratiques propres, où des acquisitions internes à une communauté circulent entre des adeptes reconnus. Qui interprète qui ici ? Les traditions scripturaires sont-elles moins instruites et connaisseuses des sociétés humaines que nos modernes sociologies? Une épistémologie ne peut se dissocier de l'histoire d'un ensemble de disciplines (voir les travaux de François Laplanche auquel l'auteur rend hommage), ni des pratiques théoriques, textuelles, sociales de ces disciplines. Quel est le statut du regard sociologue quand il considère l'activité des exégètes? On demandera: comment différenciercomparer des contenus (mythe/histoire) ; des genres d'objets (documentaire télévisé, roman, manuscrit) ; des pratiques (savants/biblistes) ; des visées (juif, protestant, catholique); et où placer tant d'innovateurs non spécialistes (Barthes, Greimas, Ricœur, Girard, Debray...) quand ils interprètent ces traditions ? Du contenu en débat dans les colloques, manuels, ateliers, des vies mêmes, on apprend peu des apports scientifiques, des hypothèses, des acquis discutés. Et comment ont-ils été produits ? Canguilhem, que cite également l'auteur, le répétait avec humour à l'âge althussérien, l'épistémologie d'une science peut être soit bourgeoise, soit prolétarienne, soit considérer le résultat final, le produit fini, soit explorer les modes et les conditions de production. L'auteur s'efforce, et c'est toute la difficulté et le risque de l'entreprise qui en font l'intérêt, de produire une enquête à la fois historienne et pragmatique, qui illustre donc de traverse ${ }^{6}$ cette " anthropologie générale des pratiques et des systèmes d'interprétation qu'appelle notre modernité », selon le vœu exprimé par Marc Augé en 1991.

\section{L'amour du texte}

On connaît l'ironie yiddish : «Dieu a créé les exégètes parce qu'il n'avait pas le temps de tout dire »... A-t-il créé les sociologues pour éclairer les premiers ? Les sciences humaines prennent-elles en partie la place, ou la fonction, naguère dévolue au religieux ? C'est selon. Mais l'hypothèse de Certeau mérite vraiment l'arrêt, pour une épistémologie/sociologie de l'exégèse. Les exégètes ont-ils entendu l'appel d'un texte ? C'est l'affaire intime de chacun, mystique au sens moderne (et non médiéval) du terme. Mais ce qu'ils partagent tous, et avec l'auteur, comme tout lecteur depuis l'aurore de la littérature, c'est certainement l'amour $d u$ texte...

Jean LAMBERT

Centre d'études interdisciplinaires des faits religieux (EHESS-CNRS) cordouan3@wanadoo.fr

6. Traverse: «Route particulière, plus courte que le grand chemin, ou menant à un lieu auquel le grand chemin ne mène pas » (Littré) ; exergue de la revue Traverses (1975-1985). 